\title{
Microglia activation in the extremely preterm human brain
}

\author{
Veena Supramaniam ${ }^{1,2}$, Regina Vontell1,3, Latha Srinivasan'2, Josephine Wyatt-Ashmead ${ }^{4}$, Henrik Hagberg ${ }^{3}$ and \\ Mary Rutherford ${ }^{1,2}$
}

BACKGROUND: The periventricular white matter (PVWM) of the immature preterm brain is selectively vulnerable to a spectrum of injury. Although essential for normal brain development, the presence of resident microglia may exacerbate PVWM injury.

METHODS: We used immunohistochemistry to investigate microglia profile in human preterm noninjured control brains and in brains with evidence of germinal matrix hemorrhage/ intraventricular hemorrhage $(\mathrm{GMH} / \mathrm{IVH})$, with median gestational age (GA) of 24.1 and $25.4 \mathrm{wk}$, respectively.

RESULTS: The number of microglia in the PVWM was higher than the other brain regions in both the control and $\mathrm{GMH}$ / IVH groups. Microglial density increased further in the PVWM of $\mathrm{GMH} / \mathrm{IVH}$ brains, regardless of hemorrhage severity and despite normal macroscopic and imaging appearances to the PVWM. This was due to an increase in activated Iba1/CD68and not Iba/CD45-immunopositive microglia. However, there were very few CD68/Ki67 colocalized cells, suggesting that the source of this increase may be due to a quick transformation of CD45-immunopositive hematopoietic microglia into CD68immunopositive microglia. There was also increased apoptosis in the PVWM of all cases of GMH/IVH, with axonal injury and increased tumor necrosis factor-a (TNF-a) expression evident in the most severe cases.

CONCLUSION: Isolated GMH/IVH may influence ongoing brain development, with a significant role played by microglial activation.

P reterm infants are at an increased risk of a spectrum of brain injuries to the white matter, which are thought to arise from a combination of inflammation, disturbance to differentiating premyelinating oligodendrocytes, astrogliosis, microglial activation, and impaired myelination (1-3). Premyelinating oligodendrocytes in the developing brain are particularly vulnerable to oxidative and excitotoxic stress $(4,5)$, and activated microglia are thought to contribute to this (6). Microglia, the resident phagocytic cells of the central nervous system (7), take on a ramified quiescent morphology in the normal mature brain, but the active forms of intermediate and amoeboid morphologies are most common in normal developing brain (8). Activated microglia in the developing white matter are involved in eliminating transcallosal projections
(9), vascularization and angiogenesis (7), myelination (10), programmed cell death, synaptic pruning (11), and axonal guidance (12).

Even though a recent study indicated that microglia exert a protective effect (13), most data suggest that they are injurious. It was proposed that the normal developmental increase in resident microglia renders the human neonatal periventricular white matter (PVWM) more susceptible to injury $(6,8,14,15)$. The PVWM regions are the most frequent site of preterm white matter injuries, such as periventricular leukomalacia, punctate lesions, and diffuse excessive high signal intensity, which are evident on magnetic resonance imaging (MRI). Animal studies have highlighted the critical role played by microglia in periventricular leukomalacia (16), lipopolysaccharide-induced inflammation $(17,18)$, neonatal hypoxic-ischemic encephalopathy $(17,19)$, and germinal matrix hemorrhage/intraventricular hemorrhage (GMH/IVH) (20). Despite a decrease in its incidence with routine use of both antenatal steroids and artificial surfactant at delivery, GMH/IVH remains one of the major complications of very preterm birth and brain injury. These neonates have an increased mortality rate (21) and display a high incidence of posthemorrhagic hydrocephalus, cerebral palsy, developmental disabilities, and cognitive impairments $(22,23)$. A recent postmortem study has shown suppressed cell proliferation and decreased numbers of premyelinating oligodendrocytes in the ganglionic eminence following preterm GMH (24), which may have an adverse effect on the premyelinating oligodendrocyte population and myelination.

The aim of this study was to investigate the microglia profile in noninjured preterm brain and to compare this profile with preterm brains with evidence of GMH/IVH. We hypothesized that activated microglia in the PVWM of the noninjured brains are abundant relative to other brain regions and that a further increase in microglia would be seen in the normal-appearing PVWM of the GMH/IVH brains.

\section{RESULTS}

Case details are shown in Table 1. In both groups, the cause of death was a result of withdrawal of care or congestive heart failure due to extreme prematurity, as reported by the perinatal pathologist. Three of the controls and three of the GMH/IVH 


\section{Articles $\mid$ Supramaniam et al.}

Table 1. Summary of clinical information

\begin{tabular}{|c|c|c|c|c|c|c|}
\hline Group and case & & Sex & $\begin{array}{c}\text { GA at } \\
\text { birth (wk) }\end{array}$ & $\begin{array}{l}\text { Postnatal } \\
\text { age }\end{array}$ & $\begin{array}{l}\text { PMA at } \\
\text { death (wk) }\end{array}$ & Clinical context \\
\hline \multicolumn{7}{|l|}{ Control } \\
\hline 2 & & M & 23.9 & $9 \mathrm{~h}$ & 23.9 & $\begin{array}{l}\text { Respiratory distress syndrome and withdrawal of care as a result of extreme } \\
\text { prematurity }\end{array}$ \\
\hline 4 & & M & 26.3 & $1 \mathrm{~d}$ & 26.4 & $\begin{array}{l}\text { Respiratory distress syndrome and congestive heart failure as a result of } \\
\text { extreme prematurity }\end{array}$ \\
\hline 5 & & $\mathrm{~F}$ & 24.1 & $4 \mathrm{~h}$ & 24.1 & $\begin{array}{l}\text { Respiratory distress syndrome and congestive heart failure as a result of } \\
\text { extreme prematurity }\end{array}$ \\
\hline 6 & & M & 23.3 & $<1 \mathrm{~h}$ & 23.3 & $\begin{array}{l}\text { Respiratory distress syndrome and congestive heart failure as a result of } \\
\text { extreme prematurity }\end{array}$ \\
\hline 8 & & $\mathrm{~F}$ & 24 & $<1 \mathrm{~h}$ & 24 & $\begin{array}{l}\text { Confirmed acute ascending amniotic fluid infection. Neonatal death due to } \\
\text { extreme prematurity }\end{array}$ \\
\hline 9 & & $\mathrm{~F}$ & 24.3 & $<1 \mathrm{~h}$ & 24.3 & $\begin{array}{l}\text { Confirmed acute ascending amniotic fluid infection. Neonatal death due to } \\
\text { extreme prematurity }\end{array}$ \\
\hline Median & & & 24.1 & $4 \mathrm{~h}$ & 24.1 & \\
\hline $\mathrm{GMH} / \mathrm{IVH}$ & Grade & & & & & \\
\hline 1 & II & $F$ & 25.4 & $1 d$ & 25.6 & $\begin{array}{l}\text { Mild hemorrhage. Respiratory distress syndrome and congestive heart } \\
\text { failure as a result of extreme prematurity }\end{array}$ \\
\hline 2 & II & $\mathrm{F}$ & 23.9 & $1 \mathrm{~d}, 16 \mathrm{~h}$ & 24 & $\begin{array}{l}\text { Mild hemorrhage. Respiratory distress syndrome and withdrawal of care as } \\
\text { a result of extreme prematurity }\end{array}$ \\
\hline 6 & IV & M & 26.3 & $4 d$ & 26.9 & $\begin{array}{l}\text { Severe hemorrhage. Respiratory distress syndrome and congestive heart } \\
\text { failure as a result of extreme prematurity }\end{array}$ \\
\hline 7 & III & M & 24 & $\begin{array}{l}4 \mathrm{wk}, 6 \mathrm{~d} \\
19 \mathrm{~h}\end{array}$ & 28.9 & $\begin{array}{l}\text { Severe hemorrhage. Confirmed acute ascending amniotic fluid infection. } \\
\text { Respiratory distress syndrome and withdrawal of care as a result of extreme } \\
\text { prematurity }\end{array}$ \\
\hline Median & & & 25.4 & $4 d$ & 26.9 & \\
\hline
\end{tabular}

Hemorrhage grade I, isolated GMH; hemorrhage grade II, intraventricular extension of hemorrhage with normal ventricle size; hemorrhage grade III, IVH with dilated ventricles; hemorrhage grade IV, grade III plus extension of GMH into adjacent brain parenchyma (3).

GA, gestational age; GMH/IVH, germinal matrix hemorrhage/intraventricular hemorrhage; PMA, postmenstrual age.

cases had confirmed amniotic fluid infection (AFI). Although the study groups were not age matched, there was no difference in the gestational age (GA) at birth or postmenstrual age at death between the two groups $(P>0.05)$. The postnatal age of the GMH/IVH group was significantly higher than that of the control group $(P<0.05)$.

There was no significant difference in the number of Iba1-, CD68-, CD45-, or caspase-3-immunopositive cells between the different lobes of the brain assessed in specific regions of interest (ROIs) (GM, PVWM, deep white matter (DWM), and cerebral cortex) in the two groups. Therefore, specific cell counts from the three lobes in an individual brain have been combined for each ROI.

In the control and GMH/IVH groups, the number of microglia in the GM and PVWM increased significantly $(P<0.05)$ with increasing postnatal age (control: GM $r^{2}=0.27$ and PVWM $r^{2}=0.47$; GMH/IVH: GM $r^{2}=0.32$ and PVWM $r^{2}$ $=0.28$ ) and postmenstrual age (control: GM $r^{2}=0.231$ and PVWM $r^{2}=0.41$; GMH/IVH: GM $r^{2}=0.62$ and PVWM $r^{2}$ $=0.2847)$. Microglia in both groups were predominantly of amoeboid and intermediate-activated morphology, with few ramified cells (amoeboid and intermediate-activated 


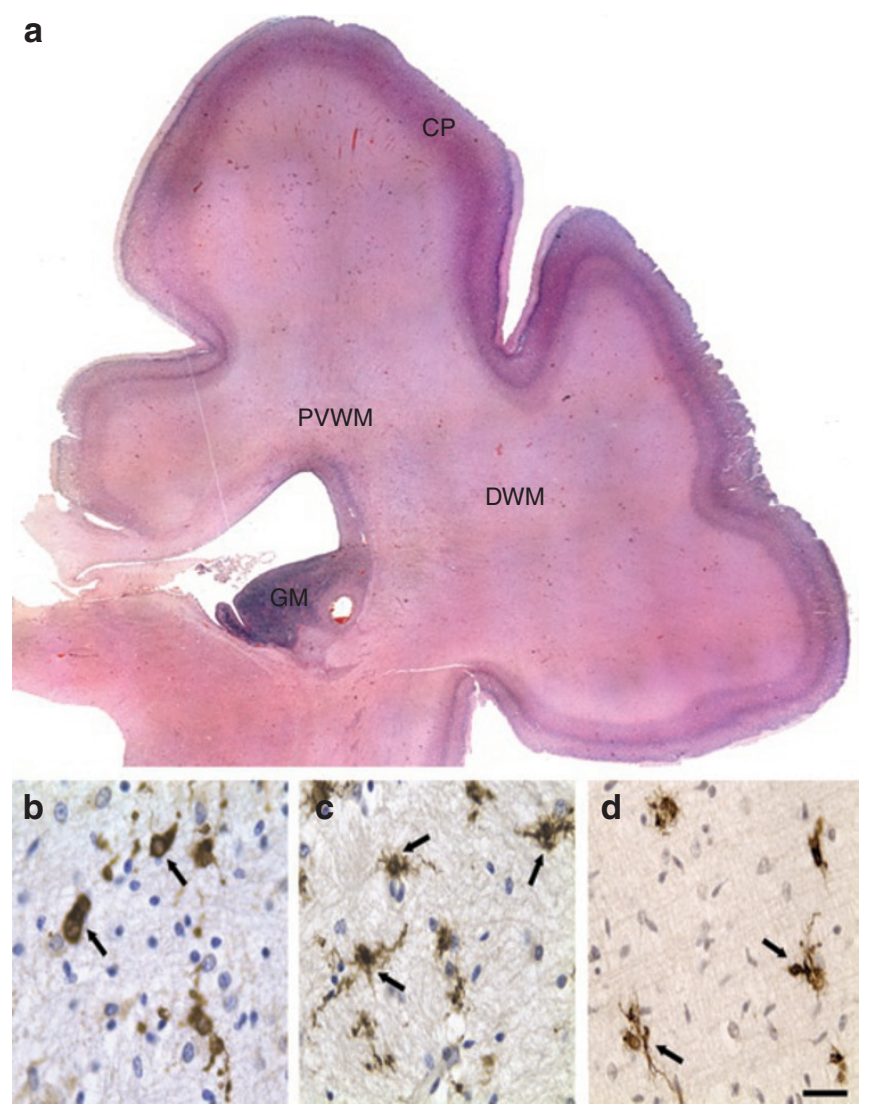

Figure 1. H\&E-stained coronal tissue section from the (a) parietal lobe of a $23^{+6 / 7}$ wk PMA control brain. Microglia morphology in the developing brain: (b) amoeboid (arrow), (c) intermediate (arrow), and (d) ramified (arrow) microglia. Bar $=25 \mu \mathrm{m}$ (applies to $\mathbf{b}-\mathbf{d}$ ). CP, cortical plate; DWM, deep white matter; GM, germinal matrix; H\&E, hematoxylin and eosin; PMA, postmenstrual age; PVWM, periventricular white matter.

microglia:ramified microglia in the GM and PVWM were 50:1 and 9:1, respectively) $(P<0.05)$. Microglia morphology is illustrated in Figure 1.

\section{Iba1-Immunopositive Microglia in the Control and GMH/IVH Groups}

In both groups (Table 2), the total number of microglia in the GM was significantly higher than in any other ROI (Figure 2b,d,e). Microglia in the PVWM was significantly higher than in the DWM and cortex (Figures $2 \mathrm{f}$ and $3 \mathrm{c}$ ). The total number and distributions of microglia in the different ROIs in the control cases with evidence of AFI were not significantly different from those without AFI $(P>0.05)$ (Table 2).

The total number of microglia in the PVWM was significantly higher in the GMH/IVH as compared with the control group $(P<0.05)$ (Table 2$)$. The study cohort had two sets of twins, with postnatal age $<2 \mathrm{~d}$, in which one twin from each set served as a control (cases no. 1 and 2) and the other was the age-matched GMH/IVH twin (cases no. 1 and 2) with mild hemorrhage (grade II) and no AFI. Of interest, the microglia number in the PVWM of the GMH/IVH twins $(124.30 \pm 2.97)$ was higher than that of the control twins $(93.53 \pm 8.22)$.
Table 2. The total number of Iba1-immunopositive microglia in the different regions of interest in the control and GMH/IVH groups

\begin{tabular}{lcc}
\hline $\begin{array}{l}\text { Region of } \\
\text { interest }\end{array}$ & $\begin{array}{c}\left.\text { Control group (cells } / \mathrm{mm}^{2}\right),(n=9) \\
(\text { with } \mathrm{AFI}(n=3) \text { vs. without AFI } \\
(n=6))\end{array}$ & $\begin{array}{c}\text { GMH/IVH group } \\
\left(\text { cells } / \mathrm{mm}^{2}\right), \\
(n=7)\end{array}$ \\
\hline GM & $\begin{array}{c}233.40 \pm 14.34 \\
\text { PVWM }\end{array}$ & $248.43 \pm 24.30$ \\
& $105.59 \pm 4.72$ & $145.81 \pm 8.74^{*}$ \\
DWM & $(110.24 \pm 5.74$ vs. $104.16 \pm 4.02)$ & \\
& $63.11 \pm 11.19$ & $68.88 \pm 7.87$ \\
Cortex & $(63.78 \pm 15.21$ vs. $61.89 \pm 17.83)$ & \\
& $33.56 \pm 13.97$ & $46.77 \pm 6.64$ \\
& $(34.25 \pm 14.66$ vs. $35.14 \pm 21.67)$ & \\
\hline
\end{tabular}

Results are expressed as mean \pm SEM

AFI, amniotic fluid infection; DWM, deep white matter; GMH/IVH, germinal matrix hemorrhage/intraventricular hemorrhage; PVWM, periventricular white matter.

*Significant difference between control and GMH/IVH group, $P<0.05$.

The total number of microglia in the PVWM of the severe GMH/IVH (grade III/IV) cases $(170.80 \pm 11.54)$ was significantly higher than that of the mild GMH/IVH (grade I/II) $(133.22 \pm 4.55)(P<0.05)$. Of note, PVWM microglia of mild $\mathrm{GMH} / \mathrm{IVH}$ was still higher than that of the control $(105.59 \pm$ 04.72) $(P<0.05)$. There was no additional effect of AFI on the number of Iba1-immunopositive microglia.

CD68- and CD45-Immunopositive Microglia and Cell Proliferation

The majority of the Ibal-immunopositive microglia in the control GM and PVWM were Iba1/CD68 colocalized rather than Iba1/CD45 colocalized $(P<0.05)$ (Figure 4$)$. There was a significant increase in the number of Iba1/CD68 colocalized microglia in the PVWM of the GMH/IVH as compared with the control group. In the GMH/IVH group, there was greater Iba1/CD68 colocalization than Iba1/CD45 colocalization in the PVWM (Figure 5). There was also a significant increase in CD45-immunopositive cells in the GM and PVWM with GMH/IVH as compared with the controls; however, a smaller proportion of these cells were Iba1/CD45 colocalized.

To address whether the increase in microglia in the PVWM of the hemorrhage brains was due to an increased proliferation of CD68-immunopositive cells, we assessed CD68/Ki67 colocalization. The results showed some CD68/Ki67 colocalization in the PVWM hemorrhage cases (Figure 6), suggesting that increased proliferation accounts for at least a small proportion of the increased numbers of CD68-immunopositive cells.

\section{Cell Apoptosis, Axonal Injury, and Cytokine Production}

The appearance of caspase-3 apoptotic cells in the PVWM $(40.91 \pm 8.74)$ of the GMH/IVH brains (Figure $2 \mathrm{~g}$ ) was significantly higher than that of the control group $(18.71 \pm 3.85)(P<$ 0.05). Apoptosis in PVWM increased with severity of hemorrhage $(48.08 \pm 2.62$ severe vs. $33.57 \pm 0.70$ mild $)$.

There was evidence of amyloid precursor protein-immunopositive axonal retraction balls, which are indicative of swollen and disconnected axons, in the PVWM of the two severe hemorrhage cases (Figure $2 \mathbf{h}$ ). These two cases also showed 

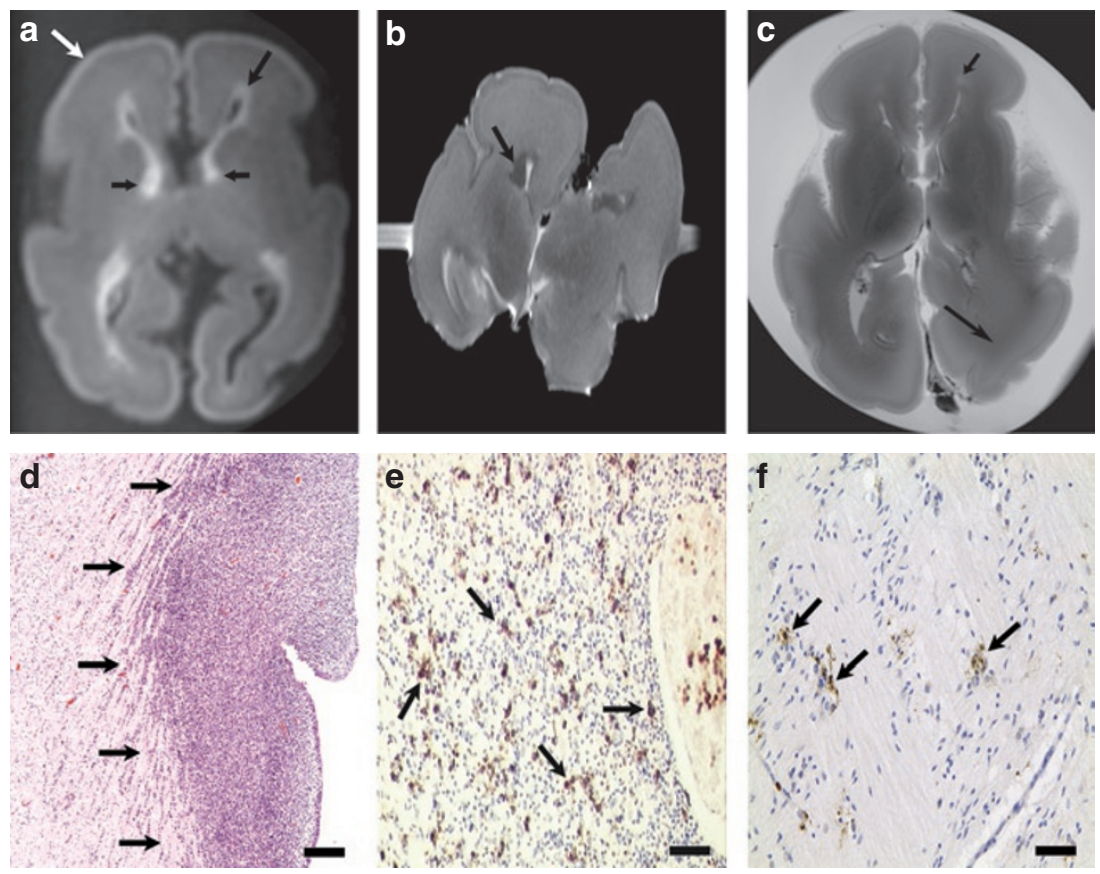

Figure 2. Postmortem MRI (axial plane) of: (a,b) control brain at $29^{+3 / 7}$ wk PMA (the fixed brain was imaged $20 \mathrm{~d}$ after death ( $16 \mathrm{~d}$ after postmortem)), and (c) control brain at $25^{+3 / 7}$ wk PMA. The fixed brain was imaged $11 \mathrm{~d}$ after death ( $10 \mathrm{~d}$ after postmortem). There was no MRI or histological evidence of pathology. The GM is shown as (a) a high signal intensity region (long black arrow) on T1-weighted image and (b) a low signal intensity region (black arrow) on T2-weighted image. The GM is most prominent in the caudothalamic notch (a, short black arrow). This corresponds to (d) a cell-dense GM region on H\&E histology (arrow) and (e) a dense population of Iba1-immunopositive microglia in the GM (arrow). (a) The cortex is shown as high signal intensity (white arrow) on T1-weighted image. The PVWM is seen as high signal intensity (short black arrow) on T2-weighted image (in c), with (f) a few Iba1-immunopositive microglia (arrow). The DWM is seen as a region of low signal intensity (long black arrow) (in c). Bar $=500 \mu \mathrm{m}$ (in d), $100 \mu \mathrm{m}$ (in e), and $50 \mu \mathrm{m}$ (in f). DWM, deep white matter; GM, germinal matrix; H\&E, hematoxylin and eosin; MRl, magnetic resonance imaging; PMA, postmenstrual age; PVWM, periventricular white matter.
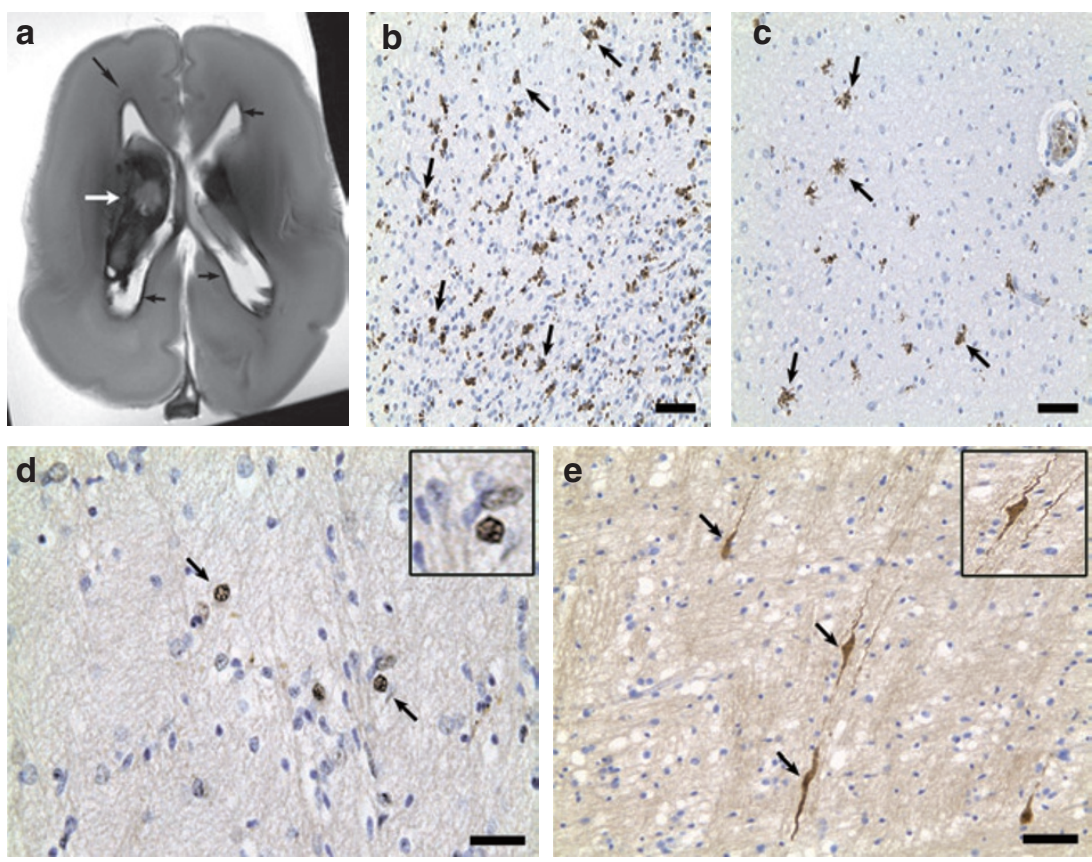

Figure 3. Postmortem MRI (axial plane) of (a) a preterm brain with multiple GMHs and a large IVH clot (white arrow) imaged at $28^{+6 / 7}$ wk PMA. Fixed brain imaged $38 \mathrm{~d}$ after death ( $36 \mathrm{~d}$ after postmortem). In addition to the focal areas of hemorrhage (white arrow), there is evidence of the characteristic marked low signal intensity lining the lateral ventricle (short black arrow) on T2-weighted image (in a). (b) This corresponded to Iba1-immunopositive microglia-rich GM (arrow). The GMH/IVH group PVWM (long black arrow) (in a) showed many (c) Iba1-immunopositive microglia (arrow), (d) caspase-3 apoptotic cells (inset), and (e) APP-immunopositive axonal retraction balls (inset). Bar $=50 \mu \mathrm{m}$ (in b, c, and e) and $25 \mu \mathrm{m}$ (in d). APP, amyloid precursor protein; GMH, germinal matrix hemorrhage; IVH, intraventricular hemorrhage; MRI, magnetic resonance imaging; PMA, postmenstrual age; PVWM, periventricular white matter. 
a

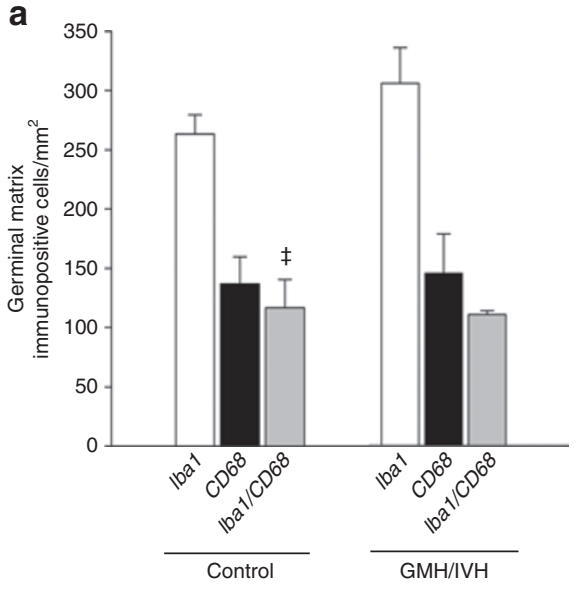

b

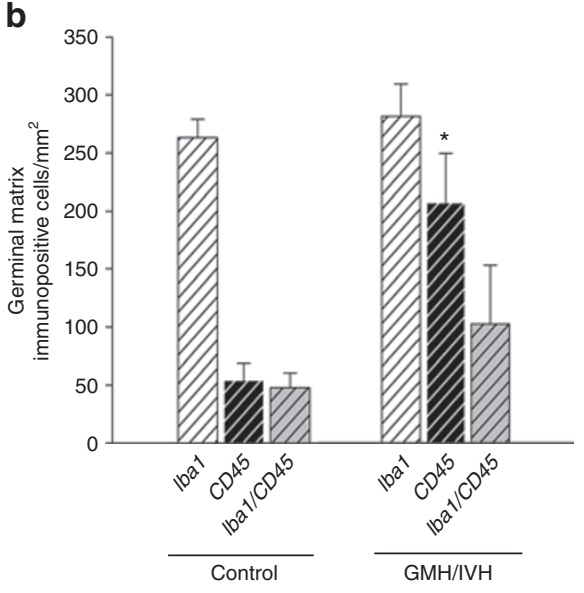

d

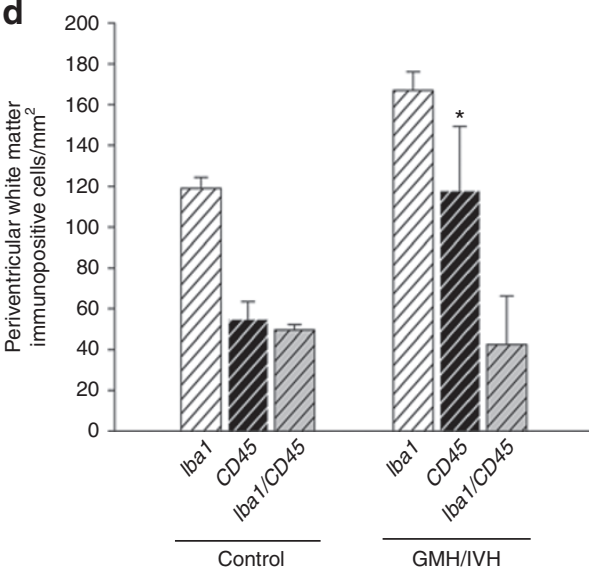

C

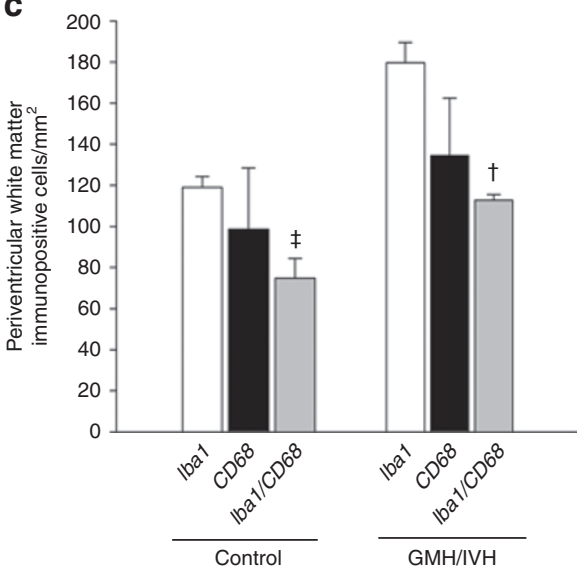

Figure 4. Quantitative analysis of cells in the GM and PVWM of the control $(n=9)$ and GMH/IVH $(n=7)$ group. Iba1 (white bar), CD68 (black bar), and Iba1/CD68 (gray bar) double-labeled cells in the (a) GM and (c) PVWM. Iba1 (white hatched bar), CD45 (black hatched bar), and Iba1/CD45 (gray hatched bar) double-labeled cells in the (b) GM and (d) PVWM. Results are expressed as mean \pm SEM and significant differences: ${ }^{\ddagger} P<0.05$ control Iba $1 / C D 68$ vs. control Iba $1-\mathrm{CD} 45 ;{ }^{*} P<0.05 \mathrm{GMH} / \mathrm{IVH}$ CD45 vs. control CD45; ${ }^{\dagger} P<0.05 \mathrm{GMH} / \mathrm{IVH}$ Iba1/CD68 vs. control Iba1/CD68. GMH, germinal matrix hemorrhage; $\mathrm{IVH}$, intraventricular hemorrhage; PVWM, periventricular white matter.

the highest incidence of caspase- 3 apoptotic cells and activated microglia in the PVWM. Of interest, there was no overt injury evident to the brain on postmortem MRI or on microscopy at conventional postmortem.

Tumor necrosis factor- $\alpha$ (TNF- $\alpha$ ) was highly expressed in the PVWM of the severe hemorrhage brain as compared with the mild hemorrhage and control groups (Figure $6 \mathbf{d}-\mathbf{i}$ ). Interleukin (IL)-10 expression was minimal in the control and GMH/IVH brains.

\section{DISCUSSION}

We have shown an increase in microglia in the PVWM as compared with other brain regions in the preterm human. This was increased further in the PVWM adjacent to GMH/IVH and was mostly due to CD68/Ibal microglia; it occurred in the presence of a normal macroscopic and MRI appearance of the PVWM. However, this increase was only partly explained by an increased proliferation of CD68 microglia. The increase in microglia in the presence of GMH/IVH was also associated with an increase in apoptotic cells, axonal injury, and enhanced microglial expression of the proinflammatory cytokine TNF- $\alpha$ in the PVWM in the most severe cases.
The large numbers of microglia in the control PVWM as compared with other areas is consistent with previous studies $(14,25,26)$, in which they were also mainly of amoeboid or intermediate active morphology. Microglia in the preterm PVWM were predominantly Iba1/CD68-immunopositive activated microglia. We demonstrated a further increase in Iba1/ CD68-immunopositive activated microglia in the PVWM in association with GMH/IVH. The more marked the severity of hemorrhage, the greater the microglia increase. Dommergues et al. (15) suggested that the increase in PVWM microglia in an excitotoxic brain injury rodent model was primarily due to proliferating resident populations of these cells. Of note, we found that a proportion of CD68-immunopositive cells expressed the marker Ki67 in the PVWM of the hemorrhage brains, indicating that proliferation contributes at least to a minor degree to the increased number of microglia. Our findings, however, support previous human studies in which a quick recruitment, migration, and transformation of microglia of hematopoietic origin to CD68-expressing microglia was suggested as being the main mechanism for the enhanced number of microglia (27). It is unclear how quickly CD45-immunopositive microglia 


\section{Articles}
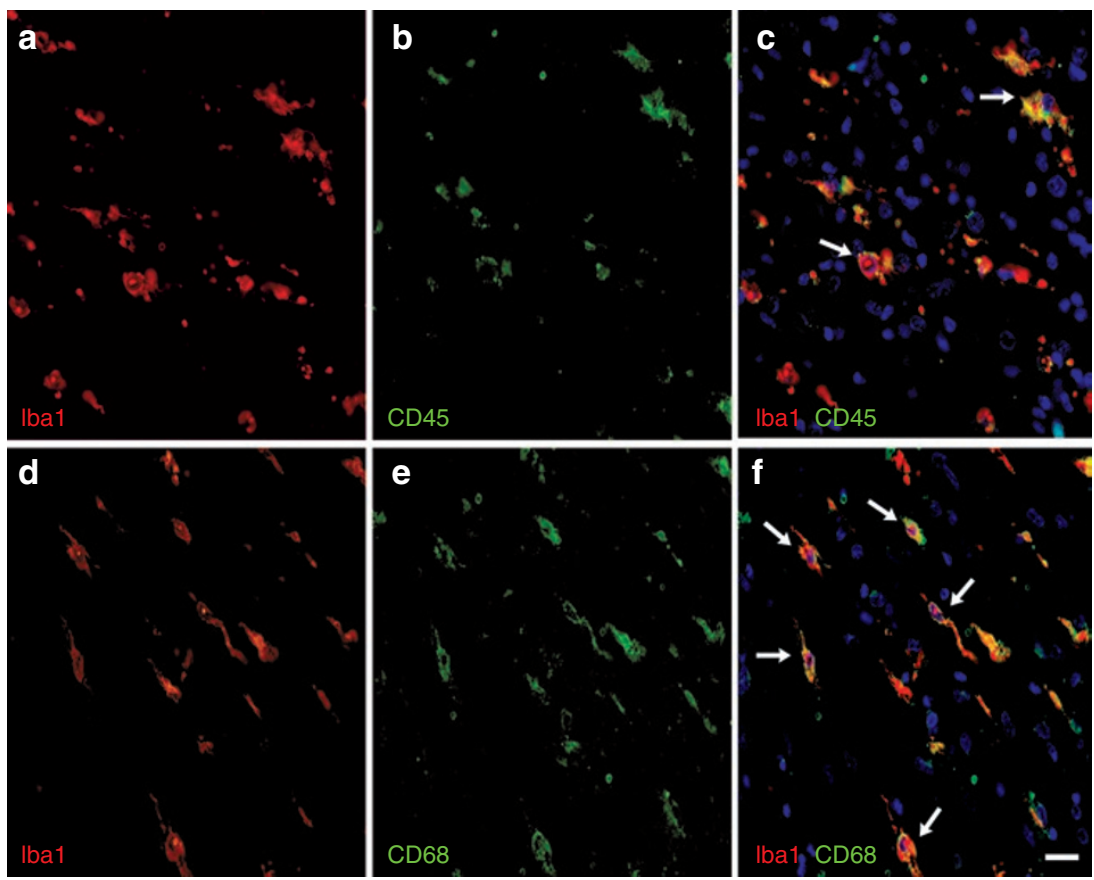

Figure 5. Immunofluorescence double labeling for (a,d) Iba1 and (b) CD45 or (e) CD68 in the PVWM of the GMH/IVH group. Colocalization of (c) Iba1/ CD45 and (f) Iba1/CD68 (white arrow) is shown in the merged image. There were more Iba1/CD68 colocalized cells than Iba1/CD45 colocalized cells. Bar = $25 \mu \mathrm{m}$ (applies to a-f). GMH, germinal matrix hemorrhage; IVH, intraventricular hemorrhage; PVWM, periventricular white matter.
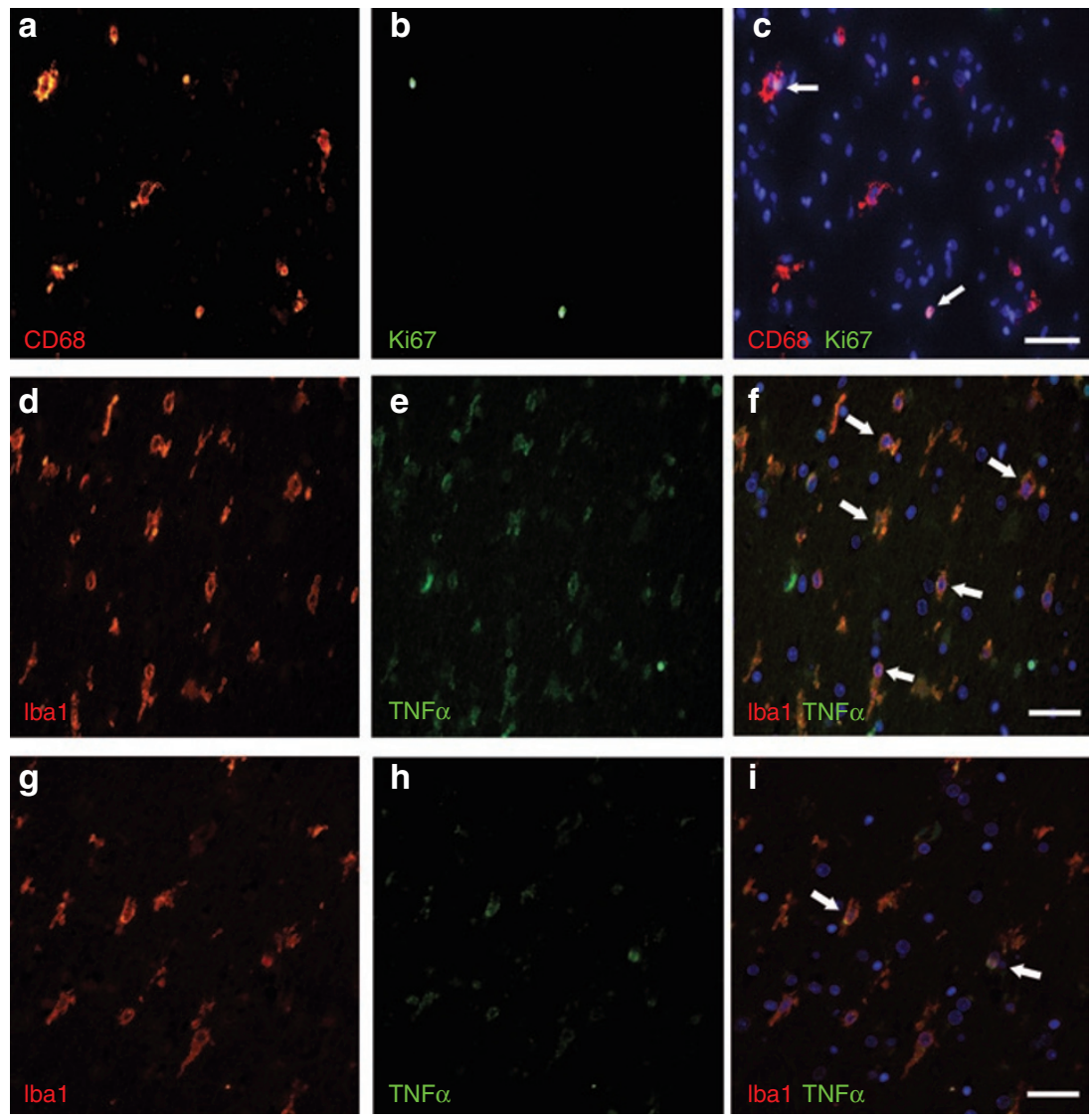

Figure 6. Immunofluorescence double labeling for (a) CD68 and (b) Ki67 in the PVWM of the GMH/IVH group. (c) Colocalization of CD68/Ki67 (white arrow) is shown in the merged image. There were some evident CD68/Ki67 colocalized cells. Immunofluorescence double labeling for Iba1 and TNF-a in the PVWM of severe $(\mathbf{d}-\mathbf{f}) \mathrm{GMH} / \mathrm{IVH}$ and $(\mathbf{g}-\mathbf{i})$ control brain showed increased colocalization of Iba1/TNF-a (white arrow) in (f) the severe as compared with (i) the control PVWM. Bar $=100 \mu \mathrm{m}$ (applies to $\mathbf{a}-\mathbf{f}$ ). GMH, germinal matrix hemorrhage; IVH, intraventricular hemorrhage; PVWM, periventricular white matter; TNF-a, tumor necrosis factor-a. 
transform into CD68-immunopositive microglia, but given the longer survival time of infants with hemorrhage, a transformation of CD45- to CD68-immunopositive microglia could have occurred. The increased microglia activation in the normal-appearing PVWM of GMH/IVH brain may involve a shift in their normal developmental role to that of immune response mediators in injury. The large number of CD45-immunopositive cells, which were not Ibal-immunopositive, in the GM and PVWM of the hemorrhagic brains may suggest an influx of lymphocytes or other hematopoietic monocytic cells to the injury site.

There was evidence of increased apoptosis in the PVWM of the hemorrhage group, and with increased severity there was increased cell apoptosis, which was associated with axonal injury in the PVWM. However, there were no imaging or histological evidence at postmortem consistent with white matter injury or infarction. To the best of our knowledge, axonal injury in association with isolated GMH/IVH that is not complicated by overt venous parenchymal infarction in the preterm neonate has not been shown previously. These findings support previous studies of a preterm rabbit model of GMH, a mouse model of IVH, and human preterm IVH in which researchers have suggested an involvement by microglia in cell apoptosis and neuronal degeneration in the PVWM and axonal injury in the DWM $(20,28)$. However, the hemorrhage in the animal models $(20,28)$ and in the preterm human brains $(20)$ were grade III and grade IV, which is more severe than in the current study. We have therefore illustrated that even in milder forms of hemorrhage (grade I/II), there is still an increased activation of microglia and cell apoptosis in the PVWM. We also showed an increased expression of the proinflammatory cytokine, TNF- $\alpha$, in PVWM of the severe hemorrhage brains, whereas the anti-inflammatory cytokine IL-10 remained unchanged. Our data support previous studies showing that the overactivation of microglia in these more severe GMH/IVH cases may be a contributing factor to the increased apoptosis and axonal injury through the release of immune-inflammatory mediators $(29,30)$. Studies have shown that blood-derived mediators from the hemorrhage, such as thrombin and plasminogen, may be involved in upregulating inflammatory cytokine release by microglia and adjacent white matter injury $(31,32)$. In the preterm neonate with GMH/IVH, white matter involvement with venous infarction may take $24-48 \mathrm{~h}$ to develop on ultrasound (33). The hemorrhage cases showing evidence of axonal injury in our study had a postnatal age $>48 \mathrm{~h}$ at the time of death and had no imaging evidence of white matter involvement, suggesting that white matter injury can occur in the absence of overt changes on conventional imaging.

Although we have demonstrated an increase in activated microglia in the normal-appearing PVWM of hemorrhage brains in this small number of human cases, we cannot disregard the fact that preterm birth itself could be a potential additional trigger for injury to the vulnerable PVWM. Moreover, the characterization of the different microglia phenotypes and their activation pathways (34) would help elucidate whether the activation of these cells has a beneficial or detrimental effect on the immature brain.
This study has also highlighted that low-field-strength MRI and conventional macroscopic postmortem examination may not be sensitive enough to diagnose the microscopic tissue damage that is observed in our cohort of preterm hemorrhage brains. Back and colleagues $(35,36)$ have more recently suggested that higher field strengths would be more sensitive in detecting histophathologically defined diffuse white matter injury, which may not be evident on conventional MRI. Although magnetic field strengths of $1.5 \mathrm{~T}$ and $3 \mathrm{~T}$ are more clinically relevant, high-field-strength MRI may be required for future postmortem studies to diagnose more subtle forms of white matter injury for comparative histological studies as seen in our cohort of preterm hemorrhage brains.

\section{METHODS}

Informed parental consent was acquired according to the guidelines of the National Health Service UK. Study ethics were obtained from the National Research Ethics Service, Hammersmith and Queen Charlotte's and Chelsea Research Ethics Services, London, UK. Sixteen extremely preterm postmortem brains $(<32 \mathrm{wk} G A)$ of vaginally delivered neonates were obtained from the Perinatal Pathology Department, Imperial Health Care Trust, London, UK.

\section{Subjects}

Nine of the cases showed no significant brain pathology on macroscopic and microscopic examination and no visible brain abnormalities on postmortem MRI. Seven of the brains showed GMH/IVH but no overt white matter abnormalities on postmortem MRI or white matter pathology at conventional autopsy (Table 1). The hemorrhage cases were classified using the Papile grading system (3), and for the purposes of this study, grades I and II were classified as mild and grade III as severe (there were no grade IV cases). Three of the controls and three of the hemorrhage cases, assessed by the pathologist, had confirmed AFI, with evidence of focal presence of neutrophils in the lung and histological evidence of umbilical funisitis, chorioamnionitis, and deciduitis. Neonates with periventricular leukomalacia, hypoxic-ischemic encephalopathy, major congenital anomalies, chromosomal defects, intrauterine death, termination, or overt culture-positive sepsis were excluded from this study.

\section{Tissue Preparation and MRI}

The bodies were refrigerated $\left(2-4^{\circ} \mathrm{C}\right)$ before postmortem examination, which was performed within $1-3 \mathrm{~d}$ of death. Whole brains after postmortem were fixed in $4 \%$ formalin before MRI; brains were fixed for $1-5$ wk depending on size. MRI was performed using a 3 Tesla Philips scanner (Philips Medical Systems, Best, The Netherlands) and an eightchannel phased array adult knee-coil with the brains suspended in $4 \%$ formalin within an air-evacuated plastic container. Images were analyzed for normal anatomical structures, the presence of GMH/IVH evidenced most readily by abnormal regions of low signal intensity on T2-weighted MRI within the GM and/or within the lateral ventricles, and any overt abnormal signal intensity within the white matter. The postmortem images demonstrated good tissue differentiation and were used to confirm normal-appearing white matter in both groups and to confirm the presence and assess the severity of hemorrhage in the GMH/IVH group. The ventricles were clearly identified as low signal intensity on T1-weighted images and high signal intensity on T2-weighted images (Figure 2a,b). The normal GM was identified as symmetrical high signal intensity on T1-weighted images and low signal intensity on T2-weighted images (Figure 2a,b) proximal to the lateral ventricles and most prominent in the caudothalamic notch (Figure 2a). The white matter showed fairly uniform low signal intensity on T1-weighted and high signal intensity on T2-weighted images. The PVWM and DWM were evident as high signal intensity and low signal intensity regions on T2-weighted (Figure 3c) and T1-weighted MRI, respectively. The cortex was identified as high signal intensity on T1-weighted image (Figure 2a). Areas of hemorrhage were seen as 
irregular regions of low signal intensity on T2-weighted postmortem MRI within the GM (Figure 3a), distorting its normal shape. A hemorrhage of low signal intensity could also be detected within the ventricles in some cases (Figure 3a).

Paraffin-embedded tissue sections from the frontal (at the level of medial frontal gyrus), parietal (at the level of postcentral gyrus including the centrum semiovale central white matter), and parietotemporal (at the level of Ammon's horn) lobes were used for immunohistochemistry.

\section{Hematoxylin and Eosin Histochemistry}

Following routine deparaffinization and hydration in water, standard hematoxylin and eosin histology staining was performed (Gill's 1 Hematoxylin and Eosin solution was purchased from Thermo Scientific, Loughborough, UK).

\section{Immunohistochemistry}

Antibodies used to investigate the microglia population were polyclonal Ibal (1:1,000; Wako Chemicals, Richmond, VA), monoclonal CD68 (kp-1 clone, 1:800; DAKO, Ely, UK), and monoclonal CD45 (LCA clone, 1:500; DAKO). All three antibodies are expressed in ramified and activated microglia (26,37). CD68 is also expressed in the lysosomal granules of macrophages in both the developing and adult brain $(38,39)$ and is a marker of phagocytosis. CD45 is expressed in nucleated hematopoietic cells and lymphocytes. Activated microglia in the first-trimester human brain preferentially express Ibal and CD45 and are suggested to be of hematopoietic leukocyte origin $(8,26,37)$. As microglia accumulate in these sites, they become more Iba1- and CD68-immunopositive $(8,26,37)$. By 25-30wk GA, Iba1immunopositive resident microglia populations in the developing human brain were strongly CD68-immunopositive and only slightly CD45-immunopositive (40). Polyclonal cleaved Caspase-3 (1:200; Cell Signalling Technology, Hitchin, UK) and monoclonal amyloid precursor protein (1:1,000; Boehringer Ingelheim, Bracknell, UK) were used to identify apoptotic cells and axonal injury, respectively. Monoclonal Ki67 (1:500; DAKO) was used to assess cell proliferation, and monoclonal TNF- $\alpha$ and IL-10 (1:100; R\&D Systems, Minneapolis, MN) were used to investigate pro- and anti-inflammatory processes, respectively. Following routine deparaffinization and hydration in water, endogenous peroxidase activity was quenched by placing the slides into $3.0 \%$ hydrogen peroxide (VWR, Leighton Buzzard, UK). The slides were immersed in preheated antigen retrieval solution, $\mathrm{pH} 6.0$ or $10 \mathrm{mmol} / \mathrm{l}$ citrate buffer pH 6.0 (VWR) before blocking in 10\% normal horse or goat serum (Vector Laboratories, Peterborough, UK) for $20 \mathrm{~min}$ and incubation overnight at $4{ }^{\circ} \mathrm{C}$ with one of the primary antibodies. This was followed by exposure to the secondary biotinylated antibodies (1:100; Vector Laboratories) horse-antimouse (monoclonal) or goat-antirabbit (polyclonal) for $1 \mathrm{~h}$, and then to avidin-biotin complex (1:200, ABC; Vector Laboratories). The reactions were visualized with the chromagen 3,3'diaminobenzidine (DAB Substrate; Sigma-Aldrich, Gillingham, UK) before light hematoxylin counterstain (VWR).

\section{Immunofluorescence}

Following the antigen retrieval step, the slides were rinsed in phosphate-buffered saline, then immersed in $200 \mathrm{mmol} / \mathrm{l}$ glycine buffer pH 7.4 (Fisher Scientific, Loughborough, UK) for $20 \mathrm{~min}$ and in phosphate-buffered saline (VWR). The blocking step was performed using a mixture of $5 \%$ normal horse serum and $5 \%$ goat serum (Vector Laboratories). The slides were incubated overnight at $4{ }^{\circ} \mathrm{C}$ in either antibody cocktail containing Iba 1/CD68 or Iba 1/CD45. This was followed by exposure of a secondary cocktail of Dylight594 antirabbit IgG and Dylight 488 antimouse IgG fluorescent antibodies (Vector Laboratories). Double immunofluorescence sequential labeling was undertaken for Ki67/CD68 or Ki67/CD45 and Iba1/TNF- $\alpha$ or Iba1/IL-10. The CSAII Tyramid Amplification System (DAKO) was used for anti-Ki67 or antiIbal, then incubated for $1 \mathrm{~h}$ followed by overnight incubation with anti-CD68, anti-TNF- $\alpha$, or anti-IL-10. Slides were rinsed with phosphate-buffered saline and incubated in Alexa546-conjugated donkeyantimouse (for Ki67) or goat-antirabbit (for Iba1) (Invitrogen, Paisley, UK). The slides were mounted using DAPI mounting medium (Vector Laboratories).

\section{Microscopy and Quantification}

The ROIs, namely the GM, PVWM, DWM, and cerebral cortex were identified and examined in the tissue samples. On the histology tissue sections stained with hematoxylin and eosin, the PVWM was identified as white matter directly adjacent to the GM with fiber orientation that was both tangential and radial, whereas the DWM was white matter in the intermediate zone (and further from the ventricle/germinal matrix) with predominantly radial fiber orientation (Figure 1). Immunopositive cells (Iba1, CD68, CD45, and caspase-3) were counted per high-power field at $\times 400$ magnification $\left(0.05073 \mathrm{~mm}^{2}\right)$ either on a Leica DM1000 LED brightfield microscope with a Leica DFC295 camera or a Leica DM6000B fluorescence microscope with a CCD color video camera and LAS software (Leica Microsystems, Milton Keynes, UK). An average of three counts was made per ROI. The high-power fields were selected on the basis of highest density of immunopositive cells identified within each ROI. In the GMH/ IVH cases, the hemorrhage itself and the cellular infiltration was nonuniform; hence, we used the same counting methods for quantification. Ki67-, TNF- $\alpha$-, and IL-10-immunopositive results were not quantified.

\section{Statistical Analysis}

All data were tested for normality using the Shapiro-Wilk parametric test. Unpaired $t$-test or Mann-Whitney $U$ nonparametric test was used to identify significant differences between the two groups for GA at birth, postnatal age, and postmenstrual age at death. Kruskal-Wallis multiple-comparison ANOVA was used to assess cell counts. Simple linear regression analysis and Pearson correlation coefficient were calculated to assess the effect of postnatal age and PMA on microglia numbers. We then accounted for any effect using analysis of covariance. Results are expressed as mean \pm SEM and statistical significance is set at $P<0.05$. Statistical analysis was performed using StatsDirect software for Windows (v.2.7; StatsDirect, Altrincham, UK).

\section{ACKNOWLEDGMENTS}

Josephine Wyatt-Ashmead assessed the postmortem cases, and Mary Rutherford analyzed the magnetic resonance images. We thank the families who consented to this study.

\section{STATEMENT OF FINANCIAL SUPPORT}

This work was supported by the Medical Research Council and the Biomedical Research Centre UK.

\section{REFERENCES}

1. Kinney HC, Back SA. Human oligodendroglial development: relationship to periventricular leukomalacia. Semin Pediatr Neurol 1998;5:180-9.

2. Back SA, Riddle A, McClure MM. Maturation-dependent vulnerability of perinatal white matter in premature birth. Stroke 2007;38:Suppl 2: 724-30.

3. Volpe JJ. Neurology of the Newborn, 5th edn. Philadelphia, PA: Saunders Elsevier, 2008:1094.

4. Volpe JJ. Brain injury in premature infants: a complex amalgam of destructive and developmental disturbances. Lancet Neurol 2009;8:110-24.

5. Miller SP, Ferriero DM. From selective vulnerability to connectivity: insights from newborn brain imaging. Trends Neurosci 2009;32:496-505.

6. Kinney HC. Human myelination and perinatal white matter disorders. J Neurol Sci 2005;228:190-2.

7. Rezaie P, Male D. Differentiation, ramification and distribution of microglia within the central nervous system examined. Neuroembryology 2002;1:29-43.

8. Verney C, Monier A, Fallet-Bianco C, Gressens P. Early microglial colonization of the human forebrain and possible involvement in periventricular white-matter injury of preterm infants. J Anat 2010;217:436-48.

9. Innocenti GM, Koppel H, Clarke S. Transitory macrophages in the white matter of the developing visual cortex. I. Light and electron microscopic characteristics and distribution. Brain Res 1983;313:39-53.

10. Hamilton SP, Rome LH. Stimulation of in vitro myelin synthesis by microglia. Glia 1994;11:326-35.

11. Paolicelli RC, Bolasco G, Pagani F, et al. Synaptic pruning by microglia is necessary for normal brain development. Science 2011;333:1456-8. 
12. Judas M, Rados M, Jovanov-Milosevic N, Hrabac P, Stern-Padovan R, Kostovic I. Structural, immunocytochemical, and mr imaging properties of periventricular crossroads of growing cortical pathways in preterm infants. AJNR Am J Neuroradiol 2005;26:2671-84.

13. Faustino JV, Wang X, Johnson CE, et al. Microglial cells contribute to endogenous brain defenses after acute neonatal focal stroke. J Neurosci 2011;31:12992-3001.

14. Billiards SS, Haynes RL, Folkerth RD, et al. Development of microglia in the cerebral white matter of the human fetus and infant. J Comp Neurol 2006;497:199-208.

15. Dommergues MA, Plaisant F, Verney C, Gressens P. Early microglial activation following neonatal excitotoxic brain damage in mice: a potential target for neuroprotection. Neuroscience 2003;121:619-28.

16. Tahraoui SL, Marret S, Bodénant C, et al. Central role of microglia in neonatal excitotoxic lesions of the murine periventricular white matter. Brain Pathol 2001;11:56-71.

17. Mallard C, Welin AK, Peebles D, Hagberg H, Kjellmer I. White matter injury following systemic endotoxemia or asphyxia in the fetal sheep. Neurochem Res 2003;28:215-23.

18. Pang Y, Campbell L, Zheng B, Fan L, Cai Z, Rhodes P. Lipopolysaccharideactivated microglia induce death of oligodendrocyte progenitor cells and impede their development. Neuroscience 2010;166:464-75.

19. McRae A, Gilland E, Bona E, Hagberg H. Microglia activation after neonatal hypoxic-ischemia. Dev Brain Res 1995;84:245-52.

20. Georgiadis $\mathrm{P}, \mathrm{Xu} \mathrm{H}$, Chua C, et al. Characterization of acute brain injuries and neurobehavioral profiles in a rabbit model of germinal matrix hemorrhage. Stroke 2008;39:3378-88.

21. Whitelaw A. Intraventricular haemorrhage and posthaemorrhagic hydrocephalus: pathogenesis, prevention and future interventions. Semin Neonatol 2001;6:135-46.

22. Murphy BP, Inder TE, Rooks V, et al. Posthaemorrhagic ventricular dilatation in the premature infant: natural history and predictors of outcome. Arch Dis Child Fetal Neonatal Ed 2002;87:F37-41.

23. Whitelaw A, Jary S, Kmita G, et al. Randomized trial of drainage, irrigation and fibrinolytic therapy for premature infants with posthemorrhagic ventricular dilatation: developmental outcome at 2 years. Pediatrics 2010;125:e852-8.

24. Del Bigio MR. Cell proliferation in human ganglionic eminence and suppression after prematurity-associated haemorrhage. Brain 2011;134(Pt 5):1344-61.

25. Kaur C, Hao AJ, Wu CH, Ling EA. Origin of microglia. Microsc Res Tech 2001;54:2-9.

26. Monier A, Adle-Biassette H, Delezoide AL, Evrard P, Gressens P, Verney C. Entry and distribution of microglial cells in human embryonic and fetal cerebral cortex. J Neuropathol Exp Neurol 2007;66:372-82.
27. Billiards SS, Haynes RL, Folkerth RD, et al. Myelin abnormalities without oligodendrocyte loss in periventricular leukomalacia. Brain Pathol 2008; 18:153-63.

28. Xue M, Balasubramaniam J, Buist RJ, Peeling J, Del Bigio MR. Periventricular/intraventricular hemorrhage in neonatal mouse cerebrum. J Neuropathol Exp Neurol 2003;62:1154-65.

29. Bal-Price A, Brown GC. Inflammatory neurodegeneration mediated by nitric oxide from activated glia-inhibiting neuronal respiration, causing glutamate release and excitotoxicity. J Neurosci 2001;21: 6480-91.

30. Brown GC, Bal-Price A. Inflammatory neurodegeneration mediated by nitric oxide, glutamate, and mitochondria. Mol Neurobiol 2003;27:325-55.

31. Juliet PA, Mao X, Del Bigio MR. Proinflammatory cytokine production by cultured neonatal rat microglia after exposure to blood products. Brain Res 2008;1210:230-9.

32. Xue M, Del Bigio MR. Injections of blood, thrombin, and plasminogen more severely damage neonatal mouse brain than mature mouse brain. Brain Pathol 2005;15:273-80.

33. Jaeger M, Grüssner SE, Omwandho CO, Klein K, Tinneberg HR, Klingmüller V. [Cranial sonography for newborn screening: a 10-year retrospective study in 11,887 newborns]. Rofo 2004;176:852-8.

34. Colton C, Wilcock DM. Assessing activation states in microglia. CNS Neurol Disord Drug Targets 2010;9:174-91.

35. Riddle A, Dean J, Buser JR, et al. Histopathological correlates of magnetic resonance imaging-defined chronic perinatal white matter injury. Ann Neurol 2011;70:493-507.

36. Buser JR, Maire J, Riddle A, et al. Arrested preoligodendrocyte maturation contributes to myelination failure in premature infants. Ann Neurol 2012;71:93-109.

37. Monier A, Evrard P, Gressens P, Verney C. Distribution and differentiation of microglia in the human encephalon during the first two trimesters of gestation. J Comp Neurol 2006;499:565-82.

38. Rezaie P, Cairns NJ, Male DK. Expression of adhesion molecules on human fetal cerebral vessels: relationship to microglial colonisation during development. Brain Res Dev Brain Res 1997;104:175-89.

39. Esiri MM, McGee JO. Monoclonal antibody to macrophages (EMB/11) labels macrophages and microglial cells in human brain. J Clin Pathol 1986;39:615-21.

40. Verney C, Pogledic I, Biran V, Adle-Biassette H, Fallet-Bianco C, Gressens P. Microglial reaction in axonal crossroads is a hallmark of noncystic periventricular white matter injury in very preterm infants. J Neuropathol Exp Neurol 2012;71:251-64. 